\title{
On the Impact of Power Allocation on Coalition Formation in Cooperative Wireless Networks
}

\author{
Mohammed W. Baidas and Allen B. MacKenzie† \\ Electrical Engineering Department, College of Engineering and Petroleum, Kuwait University, Kuwait \\ †Wireless@ Virginia Tech, Bradley Department of Electrical and Computer Engineering, Virginia Tech, Blacksburg, VA 24061 \\ (email: baidas@iiee.org, mackenab@vt.edu)
}

\begin{abstract}
In this paper, the impact of cooperative power allocation on distributed altruistic coalition formation in cooperative relay networks is studied. Particularly, equal power allocation (EPA), maxmin rate (MMR) and sum-of-rates maximizing (SRM) power allocation criteria are considered. A distributed merge-and-split algorithm is proposed to allow network nodes to form coalitions and improve their total achievable rate. The proposed algorithm is compared with that of centralized power control and coalition formation, and is shown to yield a good tradeoff between network sum-rate and computational complexity. Finally, numerical results illustrate that the SRM power allocation criterion promotes altruistic coalition formation and results in the largest coalitions among the different power allocation criteria.
\end{abstract}

Index Terms-Coalition formation, cooperation, decode-andforward (DF), network coding, power allocation

\section{INTRODUCTION}

In ad-hoc wireless networks, network nodes are independent, autonomous and selfish by nature and thus may not voluntarily share their transmission resources with other nodes. In other words, there is an element of competition and selfishness since all participating network nodes desire to maximize their utilities by maximizing their share of transmission resources. Also, randomly distributed nodes with local information may not know whom to cooperate with even if they are willing to cooperate. Although cooperative communications have been shown to yield significant performance gains [1], cooperation entails several costs such as bandwidth and power. Ignoring such costs is unwarranted as it may severely affect the nodes' own performance. Particularly, network nodes may not cooperate and instead divert their resources to direct data transmissions. Alternatively, a group of nodes could form a coalition and cooperate to maximize the overall gains of the group and thus promote altruism. Specifically, each node seeks partners to form a cooperative coalition to achieve rate improvement for itself and/or for the whole coalition. Establishing cooperation in ad-hoc wireless networks without a centralized controller is a dynamic process. In turn, designing practical distributed algorithms that can promote cooperation without relying on centralized control is a difficult but highly desirable task.

Coalitional game theory has emerged as an effective mathematical tool for modeling users' cooperation and designing distributed protocols in wireless networks. Several works have considered coalition formation for user cooperation in wireless networks. For instance, a simple distributed merge-and-split algorithm is proposed in [2] for the formation of virtual MIMO clusters of single-antenna nodes. The curse of the boundary nodes in selfish packet-forwarding wireless networks is resolved using coalitional games in [3]. In [4], fair group coalitions for power-aware routing in wireless networks is studied and distributed algorithms based on max-min fairness are proposed. Distributed coalition formations with transferable utilities and stable outcomes in relay networks are studied in [5].

In this work, altruistic coalition formation is considered and the aim is to address the following questions: (1) How can coalitions be formed in a distributed fashion?, and (2) What is the impact of different power allocation criteria on coalition formation? To form cooperative groups, a coalition formation algorithm based on merge-and-split rules is proposed and proven to converge with arbitrary merge-and-split iterations. Each network node is treated as a player, who seeks partners to form a cooperative group to improve its transmission rate and/or that of the whole group through spatial diversity while incurring some power cost to meet a target SNR for information exchange. Moreover, the impact of equal power allocation (EPA), max-min rate (MMR) and sumof-rates maximizing (SRM) power allocation criteria on coalition formation are studied. To the best of the authors' knowledge, no work has employed coalitional games in the analysis and design of protocols for altruistic coalition formation in network-coded cooperative wireless networks.

In the remainder of this paper, the system model is presented in Section II. In Section III, the coalitional formation framework is discussed, while the proposed distributed coalition formation algorithm is provided in Section IV. The different cooperative power allocation criteria are discussed in Section V, while the centralized power allocation and coalition formation are discussed in Section VI. The partition stability, convergence and complexity properties of the proposed algorithm are discussed in Section VII. Numerical results are presented in Section VIII while the conclusions are drawn in Section IX.

\section{SySTEM MODEL}

Consider an ad-hoc wireless network that consists of $N$ singleantenna half-duplex decode-and-forward nodes for $N \geq 3$ which are denoted $S_{1}, S_{2}, \ldots, S_{N}$. Each node wishes to exchange its data symbol $x_{j}$ for $j \in\{1,2, \ldots, N\}$ with a common destination $D$. The channel between nodes $S_{j}$ and $S_{i}$ is given by $h_{j, i}=e^{\jmath \theta_{j, i}} \sqrt{d_{j, i}^{-\nu}}$, with $\nu$ being the path-loss exponent and $\theta_{j, i}$ is the signal's phase uniformly distributed in the interval $[0,2 \pi]$ while $d_{j, i}$ is the distance between the two nodes. Channel $h_{j, i}$ between nodes $S_{j}$ and $S_{i}$ is assumed to reciprocal (i.e. $h_{j, i}=h_{i, j}$ ) with perfect channel estimation at each node. Also, let $\mathcal{S}=\left\{S_{1}, S_{2}, \ldots, S_{N}\right\}$ be the finite, non-empty set of all network nodes that eventually selforganize into $K$ (for $1 \leq K \leq N$ ) mutually exclusive coalitions of cooperative nodes $\mathcal{C}=\left\{C_{1}, C_{2}, \ldots, C_{K}\right\}$ with no cooperation between coalitions. Moreover, let $C_{k} \subseteq \mathcal{S}$ denote a coalition with $\left|C_{k}\right|$ nodes (where $|\cdot|$ represents the cardinality of a set) and $1 \leq\left|C_{k}\right| \leq N$. An individual non-cooperative player is called a singleton coalition while the set $\mathcal{S}$ is called the grand coalition when all the $N$ network nodes cooperate. An example of a network of $N=5$ nodes with a possible coalition formation is shown in Fig. 1. Communication between the nodes and destination is performed in a TDMA fashion over $N+1$ time-slots and is split into two phases; the broadcasting phase and the cooperation phase. 


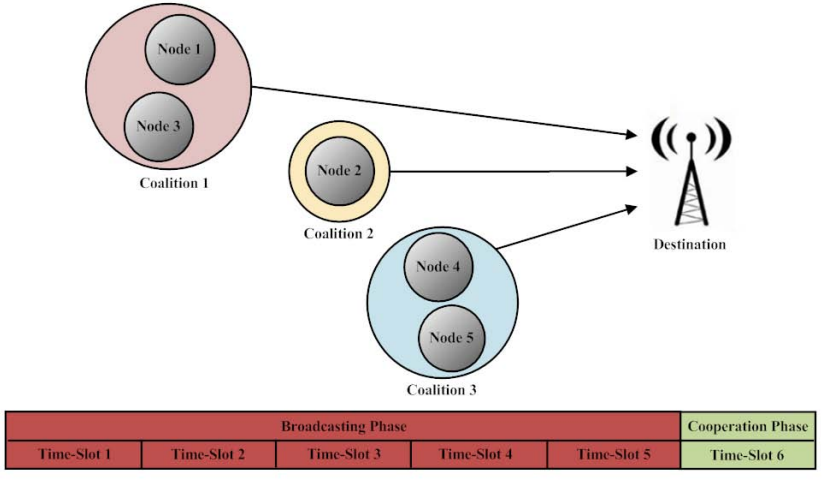

Fig. 1. Example of Cooperative Coalitions and their Transmissions

\section{A. Broadcasting Phase}

In the broadcasting phase of $N$ time-slots, each node $S_{j}$-in its assigned time-slot $T_{j}$-broadcasts its data symbol $x_{j}$, which is received by the $N-1$ other nodes $S_{i}$ in the network for $i \in$ $\{1,2, \ldots, N\}_{i \neq j}$, as well as the destination. The signal received at node $S_{i}$ for $i \neq j$ is expressed as

$$
y_{j, i}=\sqrt{P_{B_{j}}} h_{j, i} x_{j}+n_{j, i},
$$

while the received signal at the destination is given by

$$
y_{j, d}=\sqrt{P_{B_{j}}} h_{j, d} x_{j}+n_{j, d},
$$

where $P_{B_{j}}$ is the broadcasting transmit power at node $S_{j}$, and $n_{j, i}$ and $n_{j, d}$ are zero-mean complex additive white Gaussian noise (AWGN) samples with variance $N_{0}$, at node $S_{i}$ and the destination, respectively. Upon the completion of the broadcasting phase, each node $S_{i}$ will have received a set of $N-1$ signals $\left\{y_{j, i}\right\}_{j=1, j \neq i}^{N}$ comprising symbols $x_{1}, \ldots, x_{i-1}, x_{i+1}, \ldots, x_{N}$ from all the other nodes in the network, while the destination will have received $N$ signals $\left\{y_{j, d}\right\}_{j=1}^{N}$. Each node $S_{i}$ then performs a matched filtering operation on each received signal $y_{j, i}$ with signal-to-noise ratio (SNR) at the output of the matched-filter being given by [1]

$$
\gamma_{j, i}^{B P}=\frac{P_{B_{j}}\left|h_{j, i}\right|^{2}}{N_{0}}=\frac{P_{B_{j}} d_{j, i}^{-\nu}}{N_{0}} .
$$

Let $f_{j}$ denote the node farthest from $S_{j}$ in $S_{j}$ 's coalition $C_{k}$. Each node $S_{j} \in C_{k}$ broadcasts its symbol using transmit power $P_{B_{j}}$ required to maintain a target SNR of $\gamma$ between itself and node $S_{f_{j}}$ as

$$
P_{B_{j}} \geq \gamma N_{0} d_{j, f_{j}}^{\nu},
$$

where $\gamma$ is common to all the network nodes. Clearly, there is a tradeoff between the power invested in satisfying the target SNR and power allocated to the other members in coalition $C_{k}$. It should be noted that each node has a transmit power constraint of $P$ as given by $P=P_{B_{j}}+P_{C_{j}}$, where $P_{C_{j}}$ is the effective cooperative power at node $S_{j}$ to relay the symbols of the other nodes in coalition $C_{k}$. Specifically, $P_{C_{j}}$ is given by

$$
P_{C_{j}}=\max \left[0, \min \left(P-P_{B_{j}}, P\right)\right],
$$

with $P_{C_{j}}=\sum_{S_{i} \in C_{k}, i \neq j} P_{C_{i, j}}$, and $P_{C_{i, j}}$ is the cooperative power node $S_{j}$ utilizes in relaying node $S_{i}$ 's symbol $x_{i}$ to the destination.

\section{B. Cooperation Phase}

In the cooperation phase, each node $S_{i}$ for $S_{i} \in C_{k}, \forall C_{k} \in \mathcal{C}$ and $\left|C_{k}\right| \geq 2$ in time-slot $T_{N+1}$ forms a linearly-coded signal $\mathcal{X}_{i}(t)$ of the $\left|C_{k}\right|-1$ received signals from the nodes in $C_{k}$, during the broadcasting phase. For multiuser detection at the destination, each decoded symbol $x_{l}$ at node $S_{i}$ is spread using a signature waveform $c_{l}(t)$, where it is assumed that each node $S_{i}$ for $i \neq l$ and the destination know the signature waveforms of all the other nodes in the coalition. The cross-correlation of $c_{l}(t)$ and $c_{i}(t)$ is $\rho_{l, i}=\left\langle c_{l}(t), c_{i}(t)\right\rangle \triangleq\left(1 / T_{s}\right) \int_{0}^{T_{s}} c_{l}(t) c_{i}^{*}(t) d t$ for $l \neq i$ with $\rho_{i, i}=1$ and $T_{s}$ being the symbol duration. Therefore, the transmitted signal by node $S_{i}$ is expressed as

$$
\mathcal{X}_{i}(t)=\sum_{S_{l} \in C_{k}, l \neq i} \sqrt{P_{C_{l, i}}} x_{l} c_{l}(t)
$$

The received signal at the destination-assuming perfect timing synchronization-is written as

$$
\mathcal{Y}_{d}(t)=\sum_{i=1}^{N} h_{i, d} \mathcal{X}_{i}(t)+n_{d}(t)
$$

where $n_{d}(t)$ is the AWGN process at the destination. By substituting (6) into (7), $\mathcal{Y}_{d}(t)$ is re-written as

$$
\mathcal{Y}_{d}(t)=\sum_{k=1}^{K} \sum_{S_{l} \in C_{k}}\left(\sum_{S_{i} \in C_{k}, i \neq l} \sqrt{P_{C_{l, i}}} h_{i, d}\right) x_{l} c_{l}(t)+n_{d}(t) .
$$

At the destination, multiuser detection is performed on $\mathcal{Y}_{d}(t)$ to extract each symbol $x_{j}$ of node $S_{j} \in C_{k}, \forall C_{k} \in \mathcal{C}$. Specifically, $\mathcal{Y}_{d}(t)$ is passed through a matched filter bank (MFB), yielding

$$
\mathcal{Y}_{j, d}=\left\langle\mathcal{Y}_{d}(t), c_{j}(t)\right\rangle=\sum_{k=1}^{K} \sum_{S_{l} \in C_{k}} \alpha_{l} x_{l} \rho_{l, j}+\bar{n}_{j, d},
$$

where $\bar{n}_{j, d}$ is a zero-mean AWGN noise sample with variance $N_{0}$, while $\alpha_{l}$ is given by

$$
\alpha_{l}=\sum_{S_{i} \in C_{k}, i \neq l} \sqrt{P_{C_{l, i}}} h_{i, d} .
$$

It is assumed that $\rho_{l, j}=\rho, \forall l \neq j$. Thus, the decorrelated received signal is obtained as [6]

$$
\tilde{\mathcal{Y}}_{j, d}=\alpha_{j} x_{j}+\tilde{n}_{j, d},
$$

where $\tilde{n}_{j, d} \sim \mathcal{C N}\left(0, N_{0} \varrho_{N}\right)$, and $\varrho_{N}$ is given by

$$
\varrho_{N}=\frac{1+(N-2) \rho}{1+(N-2) \rho-(N-1) \rho^{2}} .
$$

The received instantaneous SNR of node $S_{j}$ 's symbol (where $S_{j} \in$ $C_{k}$ ) at the destination is given by $\gamma_{j}=\gamma_{j}^{B P}+\gamma_{j}^{C P}$, where $\gamma_{j}^{B P}$ is expressed in (3), and $\gamma_{j}^{C P}$ is obtained by passing $\tilde{\mathcal{Y}}_{j, d}$ through a matched-filter. Therefore, $\gamma_{j}$ is obtained as [7]

$$
\gamma_{j}=\frac{P_{B_{j}}\left|h_{j, d}\right|^{2}}{N_{0}}+\sum_{S_{i} \in C_{k}, i \neq j} \frac{P_{C_{j, i}}\left|h_{i, d}\right|^{2}}{N_{0} \varrho_{N}} .
$$

Upon the completion of the broadcasting and cooperation phases, the destination will have received $\left|C_{k}\right|$ independent copies of symbol $x_{j}$ of node $S_{j} \in C_{k}$ and thus achieving a diversity order of $\left|C_{k}\right|$ [1]. 


$$
\mathcal{V}\left(C_{k}\right)=\left\{\boldsymbol{v}\left(C_{k}\right) \in \mathbb{R}^{\left|C_{k}\right|} \mid \forall S_{j} \in C_{k}, v_{j}\left(C_{k}\right)=R_{j, d}^{C} \geq 0, \text { if } P_{C_{j}}>0 \text {, and } v_{j}\left(C_{k}\right)=-R_{j, d}^{D} \text {, otherwise. }\right\} .
$$

\section{COALITION FORMATION FRAMEWORK}

Let $v_{j}\left(C_{k}\right)$ denote the payoff of each node $S_{j}$ in coalition $C_{k}$. Based on the discussed system model, a singleton coalition of node $S_{j}$ occurs when it does not form a coalition with other nodes. In this case, node $S_{j}$ utilizes all its power $P$ in transmitting its data once every $N+1$ time-slots. Thus, the payoff of node $S_{j}$ is

$$
v_{j}\left(\left\{S_{j}\right\}\right)=R_{j, d}^{D}=\frac{1}{N+1} \log _{2}\left(1+\frac{P\left|h_{j, d}\right|^{2}}{N_{0}}\right),
$$

where $R_{j, d}^{D}$ is the achievable rate with direct transmission. On the other hand, for coalition $C_{k}$ with $\left|C_{k}\right| \geq 2$, the achievable rate of node $S_{j}$ due to the cooperative transmission is given by

$$
R_{j, d}^{C}=\frac{1}{N+1} \log _{2}\left(1+\frac{P_{B_{j}}\left|h_{j, d}\right|^{2}}{N_{0}}+\sum_{S_{i} \in C_{k}, i \neq j} \frac{P_{C_{j, i}}\left|h_{i, d}\right|^{2}}{N_{0} \varrho_{N}}\right) .
$$

Thus, the payoff of each node $S_{j}$ in coalition $C_{k}$ is given by $v_{j}\left(C_{k}\right)=R_{j, d}^{C}$, and the value of a coalition $C_{k}$ is given by

$$
v\left(C_{k}\right)=\sum_{S_{j} \in C_{k}} v_{j}\left(C_{k}\right)
$$

Definition 1: A coalition game is said to have a non-transferable utility (NTU) if the coalition value cannot be arbitrarily apportioned among its nodes and each node will have its own specific value within a coalition.

Based on the proposed system model, the coalition game in hand has a non-transferable utility, as a specific achievable rate for each node in a coalition is achieved.

Definition 2: A coalitional game with non-transferable utility is defined by a pair $(\mathcal{S}, \mathcal{V})$, where $\mathcal{S}$ is a finite set of $N$ players, and $\mathcal{V}$ is a set valued function such that for every coalition $C_{k} \subseteq \mathcal{S}$, $\mathcal{V}\left(C_{k}\right)$ is a closed convex subset of $\mathbb{R}^{\left|C_{k}\right|}$ that contains the payoff vectors the players in $C_{k}$ can achieve ${ }^{1}$.

In the proposed system model, $\mathcal{V}: C_{k} \rightarrow \mathbb{R}^{\left|C_{k}\right|}$ such that $\mathcal{V}(\phi)=$ $\phi$, and if $C_{k} \neq \phi$, then $\mathcal{V}\left(C_{k}\right)$ is non-empty and closed. Moreover, the coalitional set-valued function $\mathcal{V}$ of a coalition $C_{k} \subseteq \mathcal{S}$ is defined as given in (17), where $P_{C_{j}}=0$ if and only if $P_{B_{j}}=P$, which implies that $S_{j}$ has no interest in cooperation.

Remark 1: The proposed network-coded transmission model is a coalitional game $(\mathcal{S}, \mathcal{V})$ in partition form with non-transferable utility, where $\mathcal{V}\left(C_{k}\right)$ is a singleton set, as defined by (17), and is thus a closed and convex subset of $\mathbb{R}^{\left|C_{k}\right|}$.

Remark 2: In the proposed NTU coalitional game $(\mathcal{S}, \mathcal{V})$, the grand coalition rarely forms due to the target SNR power costs.

Based on the SNR target in (4), the power cost for coalition formation depends on the distance between the network nodes, which in turn governs coalitions' sizes. As the size of a coalition increases, the cooperative gain and power cost per node also increase. However, the power saving due to the diversity gains gradually diminish even with the increase in the number of cooperative nodes in a coalition, at which point, no additional nodes should join the coalition. This prevents the network nodes from forming a grand coalition and instead form independent disjoint coalitions. In turn, the proposed game is modeled as a coalition formation game, with the aim of finding the network's coalitional structure [8].

\footnotetext{
${ }^{1}$ The formulated coalition game is in the characteristic function form. That is, utilities achieved by players in a coalition are unaffected by those outside it [8].
}

IV. Distributed CoAlition Formation Algorithm

In this section, the aim is to study how coalitions can be formed in a distributed manner. Specifically, a coalition is formed if it is beneficial to at least one node in the coalition and also for the coalition as a whole. The nodes of a coalition can avoid merging with other coalitions if they are as well off as a result of not merging. Furthermore, when nodes form a coalition, they cannot unilaterally deviate on their own. In turn, coalition structure changes are determined by the members of a coalition interacting with one another as a unit. Since network nodes are rational and autonomous, the design of an iterative distributed algorithm to form a network coalition structure that improves that network sum-rate is highly desirable. But first, several concepts must be defined.

Definition 3: A collection of coalitions, denoted as $\mathcal{C}$, is defined as $\mathcal{C}=\left\{C_{1}, C_{2}, \ldots, C_{K}\right\}$ for $2 \leq K \leq N$ mutually disjoint coalitions $C_{k}$ of $\mathcal{C}$. Equivalently, a collection is any arbitrary group of disjoint coalitions $C_{k}$ of $\mathcal{C}$ that does not necessarily span all the players of $\mathcal{S}$. If a collection spans all the players in $\mathcal{S}$ (i.e. $\left.\bigcup_{k=1}^{K} C_{k}=\mathcal{S}\right)$, the collection is a partition of $\mathcal{S}$.

Definition 4: A preference operator $\triangleright$ is defined for comparing two collections $\mathcal{Q}=\left\{Q_{1}, \ldots, Q_{l}\right\}$, and $\mathcal{R}=\left\{R_{1}, \ldots, R_{p}\right\}$ that are partitions of the same subset $\mathcal{A} \subseteq \mathcal{S}$ (i.e. same players in $\mathcal{Q}$ and $\mathcal{R})$. Thus, $\mathcal{Q} \triangleright \mathcal{R}$ means that the way $\mathcal{Q}$ partitions $\mathcal{A}$ is preferred to the way $\mathcal{R}$ partitions $\mathcal{A}$.

In the coalitional game theory literature, comparison relations based on orders are split into two categories [9]: individual value orders and coalition value orders. In the former category, comparison is performed on the basis of individual payoffs (e.g. Pareto order). In the latter category, two collections (or partitions) are compared based on the value of the coalitions inside these collections, such as the utilitarian order (e.g. $\mathcal{Q} \triangleright \mathcal{R} \Longrightarrow \sum_{i=1}^{l} v\left(Q_{i}\right)>\sum_{i=1}^{p} v\left(R_{i}\right)$ ). In this work, the utilitarian order comparison relation is assumed as it is more appropriate to the studied altruistic coalition formation.

There are two successive rules for forming and breaking coalitions, known as merge-and-split rules [9].

Definition 5 (Merge Rule): Merge any collection of disjoint coalitions $\left\{Q_{1}, \ldots, Q_{l}\right\}$, where $\left\{\bigcup_{k=1}^{l} Q_{k}\right\} \triangleright\left\{Q_{1}, \ldots, Q_{l}\right\}$, thus $\left\{Q_{1}, \ldots, Q_{l}\right\} \rightarrow\left\{\bigcup_{k=1}^{l} Q_{k}\right\}$.

Definition 6 (Split Rule): Split any coalition $\left\{\bigcup_{k=1}^{l} Q_{k}\right\}$, where $\left\{Q_{1}, \ldots, Q_{l}\right\} \triangleright\left\{\bigcup_{k=1}^{l} Q_{k}\right\}$, thus $\left\{\bigcup_{k=1}^{l} Q_{k}\right\} \rightarrow\left\{Q_{1}, \ldots, Q_{l}\right\}$.

The merge-and-split rules simply mean that two (or more) coalitions will merge if their merger would do more good than harm to the overall coalition value (or equivalently, sum-rate) of the merged coalition. Otherwise, coalitions will split into smaller ones or even singletons.

\section{A. Algorithm Description}

The network operation starts at time-index $\tau=0$ with network nodes being partitioned into singleton coalitions (i.e. $C_{j}=\left\{S_{j}\right\}$ for $1 \leq j \leq N$ such that $\mathcal{C}=\left\{\left\{S_{1}\right\},\left\{S_{2}\right\}, \ldots,\left\{S_{N}\right\}\right\}$ ) and each node $S_{j}$ determines its direct transmission rate $R_{j, d}^{D}$. After that, the following phases take place.

1) Node Discovery: Each node $S_{j} \in \mathcal{S}$ discovers the neighboring potential nodes with which it can possibly merge using $P_{B_{j}}=P$. Specifically, for each node $S_{j}$, the potential partners lie 
within a circle with radius determined by the power $P \geq \gamma N_{0} d_{j, f_{j}}^{\nu}$ required for symbols' exchange while meeting the target SNR $\gamma$, as given by (4). Thus, if the received signal at node $S_{i}$ satisfies $\gamma$, it is considered to be decoded correctly. Let $\mathcal{D}_{j}$ be the set of network nodes that decoded node $S_{j}$ 's symbol correctly, i.e.

$$
\mathcal{D}_{j}=\left\{\forall S_{i} \in \mathcal{S} \text { and } i \neq j: \gamma_{j, i}^{B P} \geq \gamma\right\} \text {. }
$$

After that, node $S_{j}$ broadcasts a request-to-send (RTS) message which is received by all the nodes in $\mathcal{D}_{j}$. Then, each node $S_{i} \in \mathcal{D}_{j}$ replies to node $S_{j}$ with a clear-to-send (CTS) message that contains its CSI with the destination. If the decoding set of node $S_{j}$ is empty (i.e. $\mathcal{D}_{j}=\phi$ ), then it employs direct transmission and does not form a coalition with any other node. Otherwise, node $S_{j}$ enumerates all the possible distinct coalitions of $S_{j} \cup \mathcal{D}_{j}$. In the case of a coalition $C_{k}$, the potential nodes lie within the intersection of $\left|C_{k}\right|$ circles, each centered around node $S_{j} \in C_{k}$. Clearly, the node discovery phase significantly reduces the coalition formation space.

2) Adaptive Coalition Formation: In this phase, the time-index is updated to $\tau=\tau+1$ and each node sequentially proposes to merge with one of its potential partners. If such a merge is desirable by all the nodes according to the utilitarian order, then a coalition with one or more of the potential nodes could form by a merge agreement of all the participating nodes. For all merged coalitions, a random node is elected as a coalitionhead [10], which is responsible for periodically exchanging timing information with the rest of the coalition. After that, the power allocation fractions of each node are determined according to one of the power allocation criteria discussed in Section V. After all the nodes have made their merge decisions, the merge process ends, resulting in partition $\mathcal{M}^{\tau}=\operatorname{Merge}\left(\mathcal{C}^{\tau-1}\right)$. If the sum-rate value a group of nodes achieved by forming a coalition is less than the value achieved before the merger, they split into singletons or coalitions of smaller sizes. At the end of the split process, a partition $\mathcal{C}^{\tau}=\operatorname{Split}\left(\mathcal{M}^{\tau}\right)$ is obtained. A sequence of mergeand-split processes along with time-index updates take place in a distributed manner via appropriate control channels, depending on the achievable rate improvement of each node and coalition, until there is no need for any merging/splitting in the current partition, in which case the final partition $\mathcal{C}^{*}=\mathcal{C}^{\tau}$ is obtained.

3) Data Transmission: In this final phase, data transmission of each node takes place in the form of broadcasting and cooperation, over a total of $N+1$ time-slots and as described in Section II. Finally, the above three phases repeat in response to topology changes or mobility. The network initialization and proposed merge-andsplit coalition formation algorithm are summarized in Table I.

It should be noted that the resulting partition from the proposed merge-and-split algorithm is not guaranteed to be optimal (i.e. the one that maximizes the network sum-rate) as the formed coalitions do not exchange information about their values and thus have no way of knowing whether there are different partitions that could lead to better network sum-rate. Even if all coalition values are known, no known algorithm can determine the optimal partition with polynomial-time complexity [11].

\section{Impact of Different Power Allocation Criteria}

It is intuitive to note that network coalition formation is dependent on the cooperative power allocation within each coalition. Therefore, the following power allocation criteria are studied.
TABLE I

NETwork INITIALIZATION AND PROPOSED Distributed MERGE-AND-SPLIT COALITION FORMATION ALGORITHM

\section{Initial State:}

At the beginning of all time, initialize time-index at $\tau=0$ with the network being partitioned as $\mathcal{C}^{0}=\left\{S_{1}, S_{2}, \ldots, S_{N}\right\}$.

Coalition Formation Algorithm:

Phase 1 - Node Discovery:

Each node determines its neighbors and potential coalitions.

Phase 2 - Adaptive Coalition Formation:

Coalition formation using merge-and-split rules occurs. repeat

(a) Update time-index: $\tau=\tau+1$.

(b) $\mathcal{M}^{\tau}=\operatorname{Merge}\left(\mathcal{C}^{\tau-1}\right)$ : coalitions in $\mathcal{C}^{\tau-1}$ make merge decisions based on the merge rule.

(c) $\mathcal{C}^{\tau}=\operatorname{Split}\left(\mathcal{M}^{\tau}\right)$ : coalitions in $\mathcal{M}^{\tau}$ make split decisions based on the split rule.

until merge-and-split terminates with final partition denoted $\mathcal{C}^{*}$.

\section{Phase 3 - Data Transmission:}

Each node transmits its data symbol in the broadcasting phase and the nodes within every coalition relay data for each other during the cooperation phase.

\section{A. Equal Power Allocation (EPA)}

Under this criterion, a node $S_{i} \in C_{k}$ determines its maximum required broadcasting power as $P_{B_{i}}=\max \left\{\gamma N_{0} /\left|h_{j, i}\right|^{2}\right\}_{S_{j} \in C_{k}, j \neq i}$ and then the cooperative power $P_{C_{i}}=P-P_{B_{i}}$ is equally allocated to the other nodes in $C_{k}$ in the form of

$$
\text { (EPA) : } \quad P_{C_{j, i}}=\frac{P-P_{B_{i}}}{\left|C_{k}\right|-1}, \quad \forall S_{j} \in C_{k}, \text { and } j \neq i .
$$

\section{B. Sum-of-Rates Maximizing Power Allocation (SRM-PA)}

The sum-of-rates maximizing power allocation problem of coalition $C_{k}$ is solved by the coalition-head and is expressed as

(SRM-PA): $\max \sum_{S_{i} \in C_{k}} R_{i, d}^{C}$

$$
\begin{array}{lll}
\text { s.t. } & P_{B_{i}}+\sum_{S_{j} \in C_{k}, j \neq i} P_{C_{j, i}} \leq P, \quad \forall S_{i} \in C_{k}, \\
& P_{B_{i}} \geq \gamma N_{0} /\left|h_{j, i}\right|^{2}, & \forall S_{j}, S_{i} \in C_{k} \text { and } j \neq i, \\
& P_{B_{i}} \geq 0, & \forall S_{i} \in C_{k}, \\
& P_{C_{j, i}} \geq 0, & \forall S_{j}, S_{i} \in C_{k} \text { and } j \neq i .
\end{array}
$$

The first constraint in (20) enforces total power constraint, while the second constraint ensures the target SNR is met $\forall S_{i} \in C_{k}$. The last two constraints impose non-negativity of the allocated power.

\section{Max-Min Rate Power Allocation (MMR-PA)}

The power allocation problem under the max-min rate fairness criterion solved by the coalition-head is expressed as

(MMR-PA): $\max \eta$

$$
\begin{aligned}
& \text { s.t. } \eta-R_{i, d}^{C} \leq 0, \quad \forall S_{i} \in C_{k}, \\
& P_{B_{i}}+\sum_{S_{j} \in C_{k}, j \neq i} P_{C_{j, i}} \leq P, \quad \forall S_{i} \in C_{k}, \\
& P_{B_{i}} \geq \gamma N_{0} /\left|h_{j, i}\right|^{2}, \quad \forall S_{j}, S_{i} \in C_{k} \text { and } j \neq i, \\
& P_{B_{i}} \geq 0 \\
& P_{C_{j, i}} \geq 0 \text {, } \\
& \forall S_{i} \in C_{k} \text {, } \\
& \forall S_{j}, S_{i} \in C_{k} \text { and } j \neq i \text {. }
\end{aligned}
$$

The first constraint imposes max-min rates while the rest of the constraints are as in problem (20). Problems SRM-PA and MMRPA can be verified to be convex [12] and thus can be solved efficiently by any standard convex optimization algorithm [13]. 
TABLE II

Summary of the Number of VARIAbles and Constraints for CENTRALized Power Allocation

\begin{tabular}{|c|c|c|c|c|c|c|c|}
\hline \multirow{2}{*}{\multicolumn{2}{|c|}{ Power Allocation Criteria }} & \multicolumn{2}{|c|}{ Number of Variables } & \multicolumn{4}{|c|}{ Number of Constraints } \\
\hline & & Continuous & Binary & Max-Min & Total Power & Target SNR & Coalitions \\
\hline \multirow{2}{*}{ C-SRM-PA } & With Coalition Formation & $N^{2}$ & $\begin{array}{l}N \\
2\end{array}$ & - & $N$ & $N(N-1)$ & $F_{N}$ \\
\hline & Without Coalition Formation & $N^{2}$ & $\begin{array}{l}N \\
2\end{array}$ & - & $N$ & $N(N-1)$ & - \\
\hline \multirow{2}{*}{ C-MMR-PA } & With Coalition Formation & $N^{2}+1$ & $\begin{array}{c}N \\
2\end{array}$ & $N$ & $N$ & $N(N-1)$ & $F_{N}$ \\
\hline & Without Coalition Formation & $N^{2}+1$ & $\begin{array}{l}N \\
2\end{array}$ & $N$ & $N$ & $N(N-1)$ & - \\
\hline
\end{tabular}

Therefore, solving such problems at a coalition-head should pose no severe computational overhead.

Remark 3: Since the achievable rate of each node in a coalition $C_{k}$ is strictly monotonically increasing in the allocated power, the total power constraint is always met (i.e. $P_{B_{i}}+\sum_{S_{j} \in C_{k}, j \neq i} P_{C_{j, i}}=$ $P, \forall S_{i} \in C_{k}$ and $\left.\forall C_{k} \in \mathcal{C}\right)$.

\section{Vi. Centralized Power Allocation and Coalition FORMATION}

The centralized SRM-PA and MMR-PA criteria, namely the CSRM-PA and C-MMR-PA - with and without coalition formation constraints-are formulated as mixed-integer nonlinear programming (MINLP) problems. Let $\mathcal{I}_{j, i}$ be a binary variable defined as

$$
\mathcal{I}_{j, i}= \begin{cases}1 & \text { if nodes } S_{j} \text { and } S_{i} \text { cooperate } \\ 0 & \text { otherwise }\end{cases}
$$

\section{A. Sum-of-Rates Maximizing Power Allocation}

The centralized sum-of-rates maximizing power allocation $(\mathrm{C}$ SRM-PA) with coalition formation is formulated as

(C-SRM-PA):

$\max \frac{1}{|\mathcal{S}|+1} \sum_{S_{j} \in \mathcal{S}} \log _{2}\left(1+\frac{P_{B_{j}}\left|h_{j, d}\right|^{2}}{N_{0}}+\sum_{S_{i} \in \mathcal{S}, i \neq j} \mathcal{I}_{j, i} \frac{P_{C_{j, i}\left|h_{i, d}\right|^{2}}}{N_{0} \varrho_{N}}\right)$

$$
\text { s.t. } P_{B_{i}}+\sum_{S_{j} \in \mathcal{S}, j \neq i} \mathcal{I}_{j, i} P_{C_{j, i}} \leq P, \quad \forall S_{i} \in \mathcal{S}
$$

$$
\begin{array}{ll}
P_{B_{i}} \geq \gamma N_{0} \mathcal{I}_{j, i} /\left|h_{j, i}\right|^{2}, & \forall S_{i}, S_{j} \in \mathcal{S} \text { and } i \neq j, \\
\mathcal{I}_{j, i}-\mathcal{I}_{j, k} \mathcal{I}_{k, i} \geq 0, & \forall S_{i}, S_{j}, S_{k} \in \mathcal{S} \text { and } i \neq j \\
P_{B_{i}} \geq 0, & \forall S_{i} \in \mathcal{S}, \\
P_{C_{j, i}} \geq 0, & \forall S_{i}, S_{j} \in \mathcal{S} \text { and } i \neq j, \\
\mathcal{I}_{j, i} \in\{0,1\}, & \forall S_{i}, S_{j} \in \mathcal{S} \text { and } i \neq j .
\end{array}
$$$$
\forall S_{i}, S_{j}, S_{k} \in \mathcal{S} \text { and } i \neq j \neq k,
$$

As before, the first and second constraints impose the total power and target SNR $\gamma$ constraints are satisfied for the nodes forming coalitions, respectively. The third constraint ensures that if a node joins a coalition, then it must cooperative with all its members. The last three constraints define the range of values each decision variable can take.

\section{B. Max-Min Rate Power Allocation}

The centralized max-min rate power allocation (C-MMR-PA) with coalition formation is formulated as

(C-MMR-PA): $\max \eta$

$$
\begin{aligned}
& \text { s.t. } \eta-\frac{1}{|\mathcal{S}|+1} \log _{2}\left(1+\frac{P_{B_{j}}\left|h_{j, d}\right|^{2}}{N_{0}}+\sum_{S_{i} \in \mathcal{S}, i \neq j}^{N} \mathcal{I}_{j, i} \frac{P_{C_{j, i}}\left|h_{i, d}\right|^{2}}{N_{0} \varrho_{N}}\right) \leq 0 \text {, } \\
& \forall S_{j} \in \mathcal{S}, \\
& P_{B_{i}}+\sum_{S_{j} \in \mathcal{S}, j \neq i} \mathcal{I}_{j, i} P_{C_{j, i}} \leq P, \quad \forall S_{i} \in \mathcal{S}, \\
& P_{B_{i}} \geq \gamma N_{0} \mathcal{I}_{j, i} /\left|h_{j, i}\right|^{2} \text {, } \\
& \forall S_{i}, S_{j} \in \mathcal{S} \text { and } i \neq j, \\
& \mathcal{I}_{j, i}-\mathcal{I}_{j, k} \mathcal{I}_{k, i} \geq 0 \text {, } \\
& P_{B_{i}} \geq 0 \\
& P_{C_{j, i}} \geq 0 \\
& \mathcal{I}_{j, i} \in\{0,1\} \text {, } \\
& \forall S_{i}, S_{j}, S_{k} \in \mathcal{S} \text { and } i \neq j \neq k \text {, } \\
& \text { (24d) } \\
& \forall S_{i}, S_{j} \in \mathcal{S} \text { and } i \neq j \\
& \forall S_{i}, S_{j} \in \mathcal{S} \text { and } i \neq j \text {. }
\end{aligned}
$$

It should be noted that ignoring the coalition formation constraints under the C-SRM-PA and C-MMR-PA problems (see (23c) and (24d)) results in power allocation without any coalitional structure. In other words, if node $S_{i}$ cooperates with nodes $S_{j}$ and $S_{k}$, then nodes $S_{j}$ and $S_{k}$ do not necessarily have to cooperate. In this case, nodes $S_{i}, S_{j}$ and $S_{k}$ do not form a coalition ${ }^{2}$.

The formulated centralized optimization problems are in general NP-hard and hence cannot be solved in polynomial-time complexity [14][15]. This is because finding an optimal partition of a set $\mathcal{S}$ requires iterating over all possible partitions [8], which grows exponentially with the number of nodes in $\mathcal{S}$, as given by the well-known Bell number [16]. Moreover, the number of continuous and binary variables for the C-SRM-PA problem can be verified to be $N^{2}$ and $\left(\begin{array}{c}N \\ 2\end{array}\right)$, respectively. With respect to the C-MMR-PA problem, an additional continuous variable is required for $\eta$ along with $N$ max-min rate constraints. Moreover, $N$ and $N(N-1)$ constraints are required to enforce the total power and target SNR constraints, respectively. The number of coalition formation constraints $F_{N}$ can be determined for $N \geq 4$ as $F_{N}=N \cdot B_{N}$, where $B_{N}=\left(N-2+B_{N-1}\right)$ and $B_{3}=1$. For instance, for a network of $N=10$ nodes, the C-SRM-PA problem with coalition formation requires 145 variables and 460 constraints. Therefore, such problems can only be solved for very small-sized networks; however, for large-sized networks, the computational complexity becomes prohibitively high and is thus deemed impractical. The total number of variables and constraints required for the centralized power allocation is summarized in Table II.

\footnotetext{
${ }^{2}$ It is noteworthy that in this work, forming a coalition is defined such that the users within the coalition are closed to cooperation from users outside the coalition.
} 


\section{Partition Stability, Convergence And Complexity}

In this section, the partition stability, convergence and complexity properties of the proposed distributed coalition formation algorithm are studied.

\section{A. Partition Stability}

Stable coalition structures in coalition formation games correspond to the equilibrium state in which users do not have incentives to leave the already formed coalitions.

Definition 7 (C-Homogeneity): Given a partition $\mathcal{C}=$ $\left\{C_{1}, C_{2}, \ldots, C_{K}\right\}$, a partition $\overline{\mathcal{C}}=\left\{\bar{C}_{1}, \bar{C}_{2}, \ldots, \bar{C}_{L}\right\}$ is called $\mathcal{C}$-homogenous if for each $j \in\{1, \ldots, L\}$, some $i \in\{1, \ldots, K\}$ exists such that either $\bar{C}_{j} \subseteq C_{i}$ or $C_{i} \subseteq \bar{C}_{j}$ [17].

Based on Definition 7, any $\mathcal{C}$-homogenous partition arises from $\mathcal{C}$ by allowing each coalition either to split into smaller coalitions or to merge with other coalitions.

Definition $8\left(\mathbb{D}_{h p}(\mathcal{C})\right.$ Function): For each partition $\mathcal{C}, \mathbb{D}_{h p}(\mathcal{C})$ is the family of all $\mathcal{C}$-homogenous partitions in $\mathcal{S}$ [17]. Moreover, $\mathbb{D}_{h p}(\mathcal{C})$ allows the players to leave the partition $\mathcal{C}$ only by means of possibly multiple merges or splittings.

According to Definition $8, \mathbb{D}_{h p}(\mathcal{C})$ associates with each partition $\mathcal{C}$ of $\mathcal{S}$ the group of all partitions of $\mathcal{S}$ that the players can form through merge-and-split processes applied to $\mathcal{C}$.

Theorem $1\left(\mathbb{D}_{h p}\right.$-Stability): A partition is $\mathbb{D}_{h p}$-stable if and only if it is the outcome of iterating the merge-and-split rules [17].

Based on Theorem 1 , to find a $\mathbb{D}_{h p}$-stable partition, it suffices to iterate the merge-and-split rules starting from any initial network partition until partition $\mathcal{C}^{*}$ is reached, in which case players have no incentive to leave partition $\mathcal{C}^{*}$ through merge-and-split to form other partition in $\mathcal{S}$.

Proposition 1: The resulting final partition $\mathcal{C}^{*}$ of the proposed coalition formation algorithm is $\mathbb{D}_{h p}$-stable.

Proof: This is an immediate result of the fact that no players have an incentive to leave partition $\mathcal{C}^{*}$ through merge-and-split to form other partitions in $\mathcal{S}$.

\section{B. Convergence}

Proposition 2: Each iteration of the merge-and-split rules terminates [17].

Proposition 2 is a consequence of the fact that each iteration of the merge-and-split rules improves the network sum-rate. In turn, it guarantees that the proposed algorithm can converge.

Theorem 2: The coalition formation process based on the mergeand-split rules converges to a stable partition $\mathcal{C}^{*}$ [9].

Proof: Theorem 2 is proved by contradiction. Assume that the resulting coalition partition is not the equilibrium one and the final coalition partition is not formed yet. Then, at least two coalitions that could merge to improve their value functions. Thus, the coalition formation process must iterate through the merge-andsplit rules at least one more time until no two coalitions desire to merge, in which case the final partition $\mathcal{C}^{*}$ is reached.

\section{Communication and Computational Complexity}

The communication complexity of the proposed algorithm is related to the number of merge-and-split operations, which is directly related to the total number of coalition formation proposals $\mathcal{P}$ sent by each of the $N$ nodes. Two extreme cases are considered:
(1) if all the proposals are rejected, and (2) if all the proposals are accepted. In the first case and as described in Section IV-A, each node $S_{i} \in \mathcal{S}$ submits at most $\left|\mathcal{D}_{i}\right|$ proposals, where $\left|\mathcal{D}_{i}\right| \leq N-1$. Now, if the first node submits $N-1$ proposals and the second submits $N-2$ proposals and so on, then the total number of proposals is $\mathcal{P}_{\text {worst }}=\sum_{i=1}^{N-1} i=\frac{1}{2} N(N-1)$. Thus, in the worst case, the complexity is of the order $\mathcal{O}\left(N^{2}\right)$. In the second case where all the proposals are accepted, the total number of proposals is only $\mathcal{P}_{\text {best }}=N$, and a complexity order of $\mathcal{O}(N)$. In practice, the number of proposals is between these two extreme cases (i.e. $\mathcal{P}_{\text {best }} \leq \mathcal{P} \leq \mathcal{P}_{\text {worst }}$ ). In fact, the number of proposals is much lower than $\frac{1}{2} N(N-1)$ as the proposed algorithm tends to merge the smaller coalitions first and then the bigger ones but with reduced possibilities. Hence, if $\mathcal{L}$ messages are required per coalition formation proposal, then $\mathcal{L} \times \mathcal{P}$ messages are required until convergence of the algorithm.

An equally important factor into the operation of the distributed merge-and-split algorithm is the computational complexity involved in the cooperative power allocation. As for the EPA criterion, the calculation of power allocation at each node is trivial (i.e. with negligible computational complexity). As for the SRM-PA and MMR-PA criteria, the computational complexity is dependent on the number of nodes in each coalition (which defines the number of variables and constraints). Despite the fact that such problems are convex and can be computed efficiently, doing so repetitively may impose significant overhead and delay to each coalition-head, especially for potentially large coalitions. That is, it takes longer to compute the sum-rate of a large coalition compared to a small one. However, due to the associated power costs, most network nodes tend to form coalitions of sizes less than $N / 2$ even for dense networks, under the different power allocation criteria (as will be verified in the following Section).

\section{Simulation Results}

In the following simulations, an ad-hoc network with $N=15$ nodes is studied, where the node density varies with the square area of deployment in $\mathrm{m}^{2}$, while the destination is located at the center of the area. Moreover, the path-loss exponent is set to $\nu=3$, while the correlation coefficient is $\rho=0.40$. The total power constraint per node is $P=0.15 \mathrm{~W}$, while the noise variance is $N_{0}=10^{-5}$ W. The target SNR for information exchange is set to $\gamma=3 \mathrm{~dB}$ [1]. The simulation results are averaged over 10000 independent runs with the nodes randomly and uniformly distributed across the deployment area for different network densities.

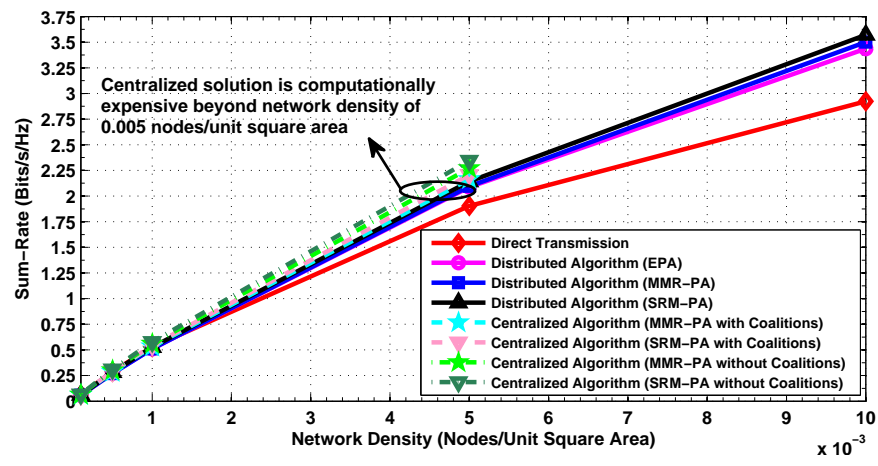

Fig. 2. Network Sum-Rate of Different Power Allocation Criteria 
It is evident from Fig. 2 that as the network density increases, the network sum-rate under the different distributed and centralized power allocation criteria also increases and is superior to that of direct transmission (which is performed over $N=15$ time-slots with no cooperation phase). This is because with the increase in network density for a fixed number of nodes, the deployment area decreases and the possibility of finding cooperative partners increases. Furthermore, the SRM-PA criterion achieves the highest sum-rate among the other power allocation criteria. Also, the centralized SRM-PA algorithm without coalition formation achieves the highest network sum-rate among all distributed and centralized power allocation criteria. Moreover, the computational complexity of the centralized algorithms for network densities beyond 0.005 nodes/unit square area becomes extremely expensive ${ }^{3}$.

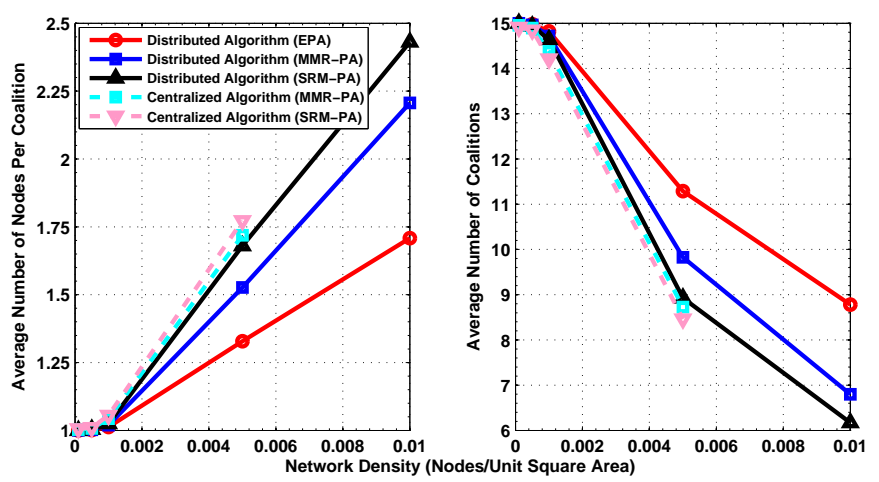

Fig. 3. Average Number of Nodes Per Coalition and Number of Coalitions of Distributed and Centralized Algorithms

From Fig. 3, it is clear that the SRM-PA criterion results in the the highest average number of nodes per coalition. This is due to the altruistic coalition formation and the fact that the SRM-PA criterion yields the highest sum-rate. Hence, network nodes tend to form larger coalitions under the SRM-PA criterion, which in turn reduces the average number of coalitions formed.

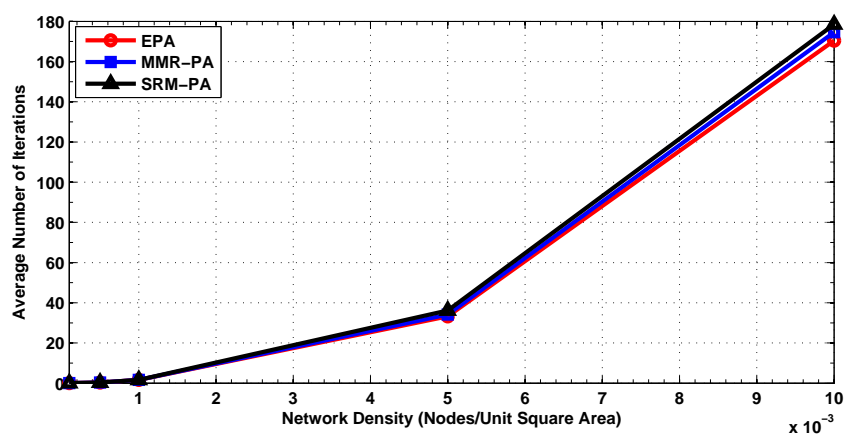

Fig. 4. Average Number of Iterations

In Fig. 4, the average number of iterations until convergence of the proposed distributed merge-and-split algorithm under the different power allocation criteria is shown. It can be seen that SRM-PA criterion requires the largest number of iterations and this is because under this criterion, network nodes tend to form larger coalitions. Thus, in the proposed distributed merge-and-split algorithm, larger potential coalitions are formed and then possibly split, which in turn increases the number of iterations.

${ }^{3}$ The centralized MINLP power allocation problems are solved using MIDACO [18] with optimization tolerance set to 0.01 .

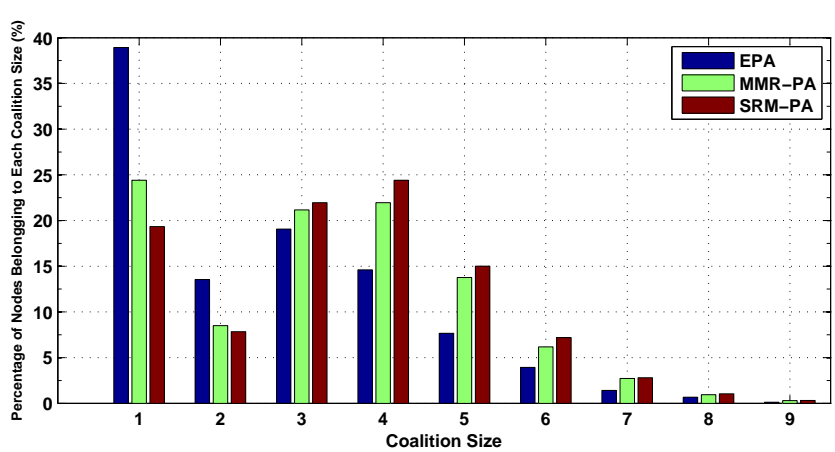

Fig. 5. Percentage of Nodes Belonging to Each Coalition Size Under the Distributed Algorithm - Network Density $=0.01$ Nodes/Unit Square Area

Based on the histogram shown in Fig. 5, it can be seen that a large portion of the nodes are participating in coalitions. Even for the EPA criterion, where singletons are most prevalent, more than half of the nodes are participating in coalitions of at least 2 nodes. As for the MMR-PA and SRM-PA criteria, more than half of the nodes are in coalitions of 3 or more nodes, with the SRM-PA criterion resulting in the largest coalitions.

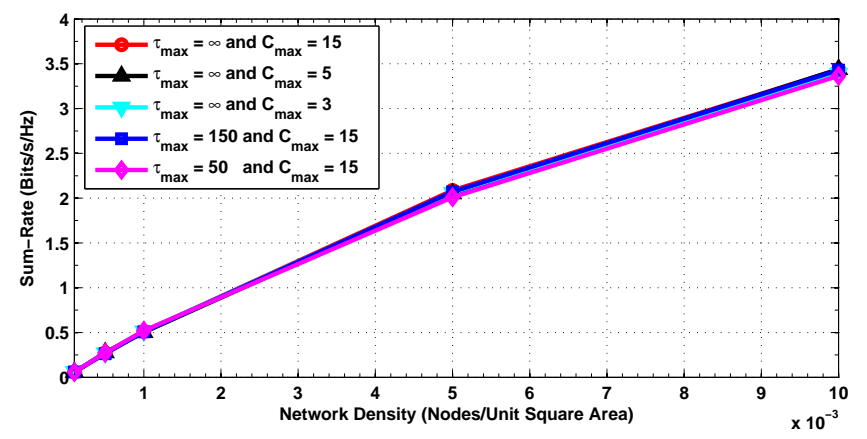

Fig. 6. Network Sum-Rate Under the Proposed Distributed Algorithm - EPA Criterion with Time-Index and Coalition Size Constraints

Based on Fig. 4, the proposed merge-and-split algorithm converge-for instance- under the EPA criterion in about 170 iterations. To allow the proposed algorithm to converge faster and reduce the communication and computational complexities, the algorithm time-index can be set to a maximum value of $\tau_{\max }$. An alternative method to speed up the convergence of the proposed algorithm is to restrict the maximum coalition size to $C_{\max }$. Fig. 6 shows that by reducing the value of $\tau_{\max }$ without restricting the coalition size (i.e. $C_{\max }=15$ ), the degradation in the sumrate is insignificant, even for $\tau_{\max }=50$. Similarly, by reducing the maximum coalition size without capping the algorithm timeindex (i.e. $\tau_{\max }=\infty$ ), the sum-rate marginally degrades. In Fig. 7 , the average number of iterations for different combinations of time-index and coalition size restrictions is illustrated. Evidently, such constraints significantly reduce the number of iterations at the expense of negligible reduction in the network sum-rate.

Finally, Fig. 8 shows the percentage of nodes belonging to each coalition size under the different constraints. It is evident that decreasing the values of $\tau_{\max }$ and $C_{\max }$ prevents large coalitions from forming, which in turn increases the percentage of nodes remaining as singletons and decreases the percentage of nodes forming coalitions of larger sizes. 


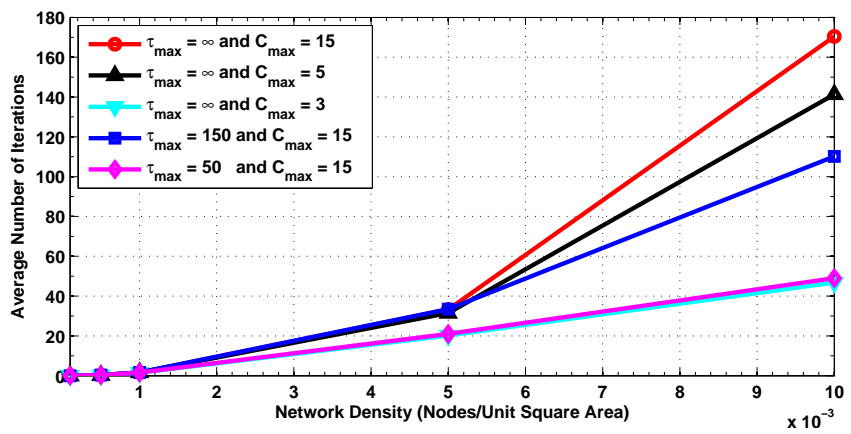

Fig. 7. Average Number of Iterations Under the Proposed Distributed Algorithm - EPA Criterion with Time-Index and Coalition Size Constraints

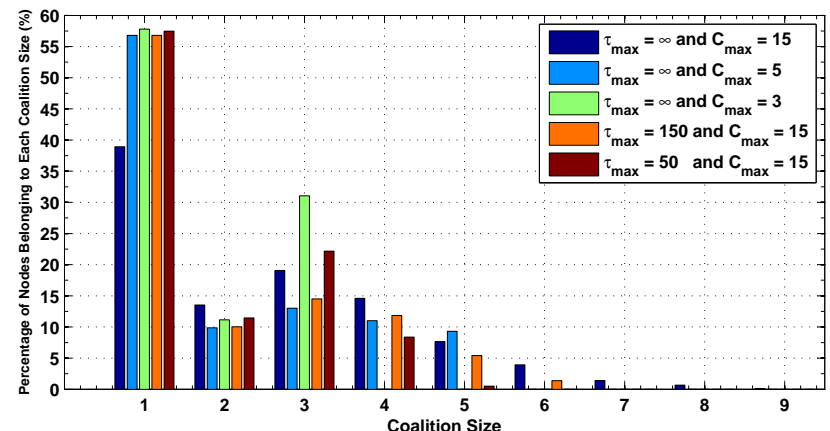

Fig. 8. Percentage of Nodes Belonging to Each Coalition Size Under the Proposed Distributed Algorithm - EPA Criterion with Time-Index and Coalition Size Constraints - Network Density $=0.01$ Nodes/Unit Square Area

\section{CONCLUSIONS}

In this paper, the problem of altruistic coalition formation in cooperative ad-hoc wireless networks is investigated. In particular, a coalition game-theoretic framework is applied to the study of distributed altruistic coalition formations to improve the overall network sum-rate. In turn, a distributed merge-and-split algorithm has been designed based on the utilitarian order and evaluated under different power allocation criteria. It has been shown that the proposed algorithm allows the network to self-organize into disjoint coalitions and that the sum-of-rates maximizing power allocation criterion results in the largest average coalition size and number of nodes per coalition, among the different power allocation criteria. Finally, the proposed algorithm yields a network sum-rate that is comparable with that of centralized control; however, with less computational complexity.

\section{REFERENCES}

[1] K. J. R. Liu, A. K. Sadek, W. Su, and A. Kwasinski, Cooperative Communications and Networking. Cambridge University Press, 2008

[2] W. Saad, Z. Han, M. Debbah, and A. Hjorungnes, "A distributed coalition formation framework for fair user cooperation in wireless networks," IEEE Trans. on Wireless Comms, vol. 8, pp. 4580 - 4593, Sept. 2009.

[3] Z. Han and H. V. Poor, "Coalition games with cooperative transmission: A cure for the curse of boundary nodes in selfish packet-forwarding wireless networks," IEEE Trans. on Comms., vol. 57, pp. 203 - 213, Jan. 2009.

[4] R. K. Guha, C. A. Sarkar, C. Singh, and A. Kumar, "Fair coalitions for poweraware routing in wireless networks," IEEE Trans. on Mobile Computing, vol. 6, pp. 206 - 220, Feb. 2007.

[5] A. Mukherjee and H. M. Kwon, "A coalition game framework for decode-andforward relay networks," Proc. of IEEE 70th Vehicular Technology Conference (VTC), pp. $1-5$, Sept. 2009.
[6] M. W. Baidas and A. B. MacKenzie, "Space-time network coding with optimal node selection for amplify-and-forward cooperative networks," Proc. of IEEE Consumer Comms. and Networking Conf., pp. 1202 - 1206, Jan. 2011.

[7] J. Luo, R. S. Blum, L. J. Cimini, L. J. Greenstein, and A. M. Haimovich, "Decode-and-forward cooperative diversity with power allocation in wireless networks," IEEE Trans. on Wireless Comms., vol. 6, no. 3, pp. 793 - 799, Mar. 2007.

[8] W. Saad, Z. Han, M. Debbah, A. Hjrungnes, and T. Basar, "Coalitional game theory for communication networks: A tutorial," IEEE Signal Processing Magazine, vol. 26, no. 5, pp. 77 - 97, Sept. 2009.

[9] K. Apt and A. Witzel, "A generic approach to coalition formation (extended version)," Int. Game Theory Rev., vol. 11, no. 3, pp. 347 - 367, Mar. 2009.

[10] A. Scaglione, D. Goeckel, and J. N. Laneman, "Cooperative communications in mobile ad-hoc networks: Rethinking the link abstraction," IEEE Signal Processing Magazine, vol. 23, pp. 18-29, Sept. 2006.

[11] G. J. Woeginger, "Exact algorithms for NP-hard problems: A survey," Combinatorial Optimization, vol. 2570, pp. 185 - 207, 2003.

[12] K. T. Phan, L. B. Le, S. A. Vorobyov, and T. L. N. Ngoc, "Power allocaion and admission control in multiuser relay networks via convex programming: Centralized and distributed schemes," EURASIP Journal on Wireless Communications and Networking, 2009.

[13] S. Boyd and L. Vandenberghe, Convex Optimization. Cambridge University Press, 2003.

[14] P. Bonami, M. Kilinc, and J. Linderoth, "Algorithms and software for convex mixed integer nonlinear programs," Technical Report \# 1664, Computer Sciences Department, University of Wisconsin-Madison, 2009.

[15] I. Nowak, Relaxation and Decomposition Methods for Mixed Integer Nonlinear Programming. Springer Scienece and Business Media - Birkhauser Verlag, Basel, 2005.

[16] T. Sandholm, K. Larson, M. Anderson, O. Shehory, and F. Tohme, "Coalition structure generation with worst case guarantees," Artificial Intelligence, vol. 10, pp. $209-238$, Jul. 1999.

[17] K. Apt and T. Radzik, "Stable partitions in coalitional games," 2006, Online: http://arxiv.org/abs/cs.GT/0605132.

[18] M. Schluter, M. Gerdts, and J. J. Ruckmann, "MIDACO: New global optimization software for MINLP," 2011, Online: http://www.midaco-solver. com/download_files/MIDACO_Paper.pdf. 